\title{
Representations of Graphs Modulo $n$
}

\author{
Anthony B. Evans* Garth Isaak ${ }^{\dagger}$ Darren A. Narayan
}

\begin{abstract}
A graph is said to be representable modulo $n$ if its vertices can be labelled with distinct integers between 0 and $n-1$ inclusive such that two vertices are adjacent if and only if their labels are relatively prime to $n$. The representation number of graph $G$ is the smallest $n$ representing $G$. We review known results and investigate representation numbers for several new classes. In particular, we relate the representation number of the disjoint union of complete graphs to the existence of complete families of mutually orthogonal Latin squares.
\end{abstract}

\section{Introduction}

For a finite graph $G$, with vertices $\left\{v_{1}, \ldots, v_{r}\right\}$, a representation of $G$ modulo $n$ is a set $\left\{a_{1}, . ., a_{r}\right\}$ of distinct, nonnegative integers, $0 \leq a_{i}<n$ satisfying $\operatorname{gcd}\left(a_{i}-a_{j}, n\right)=1$ if and only if $v_{i}$ is adjacent to $v_{j}$. The representation number, $\operatorname{Rep}(G)$, is the smallest $n$ such that $G$ has a representation modulo $n$. It was shown by Erdös and Evans [1] that any finite graph can be represented modulo some positive integer, and so the representation number of a finite graph is well defined.

We will survey known results and techniques for determining representation numbers. The representation number of a graph is related to its product dimension as defined by Nešetřil and Pultr [8]. Reviewing work on both of these problems we can observe new results in each case. We will also obtain some new results on representation numbers for certain graphs classes. These include complete multipartite graphs and graphs whose complements are paths, cycles and stars along with isolated

${ }^{*}$ Department of Mathematics and Statistics, Wright State University, Dayton, OH 45434 tevans@math.wright.edu

${ }^{\dagger}$ Department of Mathematics, Lehigh University, Bethlehem, PA 18015 gisaak@lehigh.edu Partially supported by a grant from the Reidler Foundation

${ }^{\ddagger}$ Department of Mathematics, Lehigh University, Bethlehem, PA 18015 dan6@lehigh.edu Partially supported by NSF REU program at the University of Dayton 
vertices. One of our main results illustrates the difficulty of determining representation numbers and product dimension by showing that the representation numbers of disjoint unions of complete graphs are closely related to the existence of complete families of mutually orthogonal Latin squares.

Note that throughout this paper we will use the symbol $p_{i}$ to denote the $i$-th prime; and for a graph $G$ we will use $N(v)$ to denote the open neighbourhood of a vertex $v$ of $G$, and $G^{C}$ to denote the complement of $G$. In addition let $G+H$ be the graph with vertex set $V(G) \cup V(H)$ and edge set $E(G) \cup E(H)$, and let $G \vee H$ be the graph with vertex set $V(G) \cup V(H)$ and edge set $E(G) \cup E(H) \cup\{(x, y), x \in V(G), y \in V(H)\}$. In each case we assume that $V(G)$ and $V(H)$ are disjoint. The sum $G+G \cdots+G$ of $m$ copies of $G$ will be written $m G$. For $V(H) \subseteq V(G)$ and $E(H) \subseteq E(G)$ let $G-H$ denote the graph on $V(G)$ with $E(G-H)=E(G)-E(H)$. We will almost always have $G$ a complete graph, with $K_{m}-H$ the graph whose complement is $H$ along with $m-|V(H)|$ isolated vertices. We will use $P_{n}$ and $C_{n}$ to denote paths and cycles on $n$ vertices respectively and $K_{s_{1}, s_{2}, \ldots, s_{t}}$ for the complete multipartite graph with $t$ parts having sizes $s_{1}, s_{2}, \ldots, s_{t}$. For any undefined notation see West [11].

\section{General Theory}

In this section we will review results involving the numbers and sizes of primes in the factorization of representation numbers. These tools will be used subsequently in determining representations and representation numbers of graphs and classes of graphs.

Of the many types of graph products, one is of particular interest to us: the Kronecker Product, $G \times{ }_{K} H$ of graphs $G$ and $H$ is the graph with vertex set $\{(u, v): u$ a vertex of $G, v$ a vertex of $H\},\left(u_{1}, v_{1}\right)$ being adjacent to $\left(u_{2}, v_{2}\right)$ if and only if $u_{1}$ is adjacent to $u_{2}$ in $G$ and $v_{1}$ is adjacent to $v_{2}$ in $H$, where adjacency excludes equality. Note that loops are disallowed. The Kronecker product is so named because the adjacency matrix of $G \times_{K} H$ is the Kronecker product of the adjacency matrix of $G$ and the adjacency matrix of $H$. Other names for this product can be found in literature; the tensor product, the categorical product, the cardinal product, or simply the product as in Lovász, Nešetřil, and Pultr [6] and Nešetřil, and Pultr [8].

A graph $G$ is reduced if no two vertices of $G$ have the same open neighbourhood, and a reduction of $G$ is any reduced graph obtained from $G$ by repeatedly identifying pairs of vertices with common open neighbourhoods. As any two reductions of $G$ are isomorphic, we will use the phrase "the reduction of $G$ " instead of "a reduction of $G$ ". For $v$ a vertex of $G$ we will define the reduction number, red $(v)$, of $v$ to be $|\{u \in G: N(u)=N(v)\}|$, and the reduction number, $\operatorname{red}(G)$, of $G$ to be $\max \{\operatorname{red}(v)$ : $v \in G\}$. We will say that $G$ has a constant reduction number $c$ if $\operatorname{red}(v)=c$ for all 
vertices $v$ of $G$. A graph with constant reduction is uniquely determined, up to isomorphism, by its reduction and its reduction number.

If we use $G_{n}$ to denote the graph with representation $\{0, \ldots, n-1\}$ modulo $n$ then it is obvious that $\operatorname{Rep}\left(G_{n}\right)=n$ and that a graph $H$ is representable modulo $n$ if and only if $H$ is isomorphic to an induced subgraph of $G_{n}$.

Let us look at the structure of $G_{n}$. Assume that $n=p_{i_{1}}^{k_{1}} \cdots p_{i_{m}}^{k_{m}}$, where $p_{i_{1}}, \ldots, p_{i_{m}}$ are distinct primes. If in the representation of $G_{n}$ a vertex $v$ corresponds to an integer $a$ then we will assign coordinates to $v$ as follows. The coordinates of $v$ with respect to the ordered set of primes $p_{i_{1}}, \ldots, p_{i_{m}}$ are $\left(v_{1}, \ldots, v_{m}\right)$, where $v_{j} \equiv a \bmod p_{i_{j}}$ for $j=1, \ldots, m$, and $v_{j} \in\left\{0, \ldots, p_{i_{j}}-1\right\}$ for $j=1, \ldots, m$. If $u$ has coordinates $\left(u_{1}, \ldots, u_{m}\right)$ and $v$ has coordinates $\left(v_{1}, \ldots, v_{m}\right)$ then $u$ is adjacent to $v$ if and only if $u_{j} \neq v_{j}$ for $j=1, \ldots, m$. In this coordinate representation two vertices of $G_{n}$ share the same coordinates if and only if they share the same neighbourhood. Thus we know the structure of $G_{n}$.

Lemma 2.1 If $n=p_{i_{1}}^{k_{1}} \cdots p_{i_{m}}^{k_{m}}$ where $p_{i_{1}}, \ldots, p_{i_{m}}$ are distinct primes, then $G_{n}$ has constant reduction number $p_{i_{1}}^{k_{1}-1} \cdots p_{i_{m}}^{k_{m}-1}$ and reduction $K_{p_{i_{1}}} \times_{K} \cdots \times_{K} K_{p_{i_{m}}}$.

Proof. See Theorem 2.1 in Evans, Fricke, Maneri, McKee, and Perkel [4].

We next partially answer the question of embeddability of $G_{n}$ in $G_{m}$.

Lemma 2.2 If $n=p_{i_{1}}^{k_{1}} \cdots p_{i_{s}}^{k_{s}}$ and $m=p_{j_{1}}^{r_{1}} \cdots p_{j_{t}}^{r_{t}}$, where $p_{i_{1}}<\cdots<p_{i_{s}}$ and $p_{j_{1}}, \ldots p_{j_{t}}$ are sets of distinct primes, $t \geq s, p_{j_{l}} \geq p_{i_{l}}$ for $l=1, \ldots, s, p_{j_{l}}>p_{i_{1}}$ for $l=s+1, \ldots, t$, and $p_{i_{1}}^{k_{1}-1} \cdots p_{i_{s}}^{k_{s}-1} \leq p_{j_{1}}^{r_{1}-1} \cdots p_{j_{t}}^{r_{t}-1}$, then $G_{n}$ can be embedded in $G_{m}$.

Proof. Lemma 2.1 leaves us to only consider the case in which both $n$ and $m$ are square-free. In this case the map $\left(c_{1}, \ldots, c_{s}\right) \rightarrow\left(c_{1}, \ldots, c_{s}, c_{1}, \ldots, c_{1}\right)$ embeds $G_{n}$ into $G_{m}$.

Note that $G_{n}$ might be embeddable in $G_{m}$ even if $\operatorname{red}\left(G_{n}\right)>\operatorname{red}\left(G_{m}\right)$. We can now state the following which is a stronger form of Lemma 2.4 in Evans, Fricke, Maneri, McKee, and Perkel [4].

Theorem 2.3 (i) If a graph $G$ is representable modulo $p_{i_{1}}^{k_{1}} \cdots p_{i_{m}}^{k_{m}}$, where $p_{i_{1}}<\cdots<$ $p_{i_{m}}$ are primes, then the reduction of $G$ is representable modulo $p_{i_{1}} \cdots p_{i_{m}}$.

(ii) If the reduction of a graph $G$ is representable modulo $p_{i_{1}} \cdots p_{i_{m}}$, where $p_{i_{1}}<\cdots<$ $p_{i_{m}}$ are primes, and $p_{j_{1}}, \ldots, p_{j_{n}}$ are distinct primes, $n \geq m, p_{j_{t}} \geq p_{i_{t}}$ for $t=1, \ldots, m$, $p_{j_{t}} \geq p_{i_{1}}$ for $t=m+1, \ldots, n$, and the reduction number of $G$ is at most $p_{j_{1}}^{k_{1}-1} \cdots p_{j_{n}}^{k_{n}-1}$ then $G$ is representable modulo $p_{j_{1}}^{k_{1}} \cdots p_{j_{n}}^{k_{n}}$. 
Another way of viewing this is that if $\operatorname{Rep}(G)=p_{i_{1}}^{k_{1}} p_{i_{2}}^{k_{2}} \cdots p_{i_{s}}^{k_{s}}$ then $G$ can be embedded into an $s$ dimensional array with dimensions $p_{i_{1}} \times p_{i_{2}} \times \cdots \times p_{i_{s}}$ with at most $\prod_{j=1}^{s} p_{i_{j}}^{k_{j}-1}$ vertices per cell such that vertices in the same cell form an independent set and such that vertices in different cells are non adjacent if and only if their cells are contained in a subarray of smaller dimension. Viewing the array 'geometrically' non-adjacency occurs if the cells are in some 'hyperplane'.

In the above we have viewed representation in two different ways. Given a graph $G$ and a representation $\left\{a_{1}, \ldots, a_{m}\right\}$ modulo $n$ of $G$, we may also represent $G$ by listing the coordinates with respect to $p_{i_{1}}, \ldots, p_{i_{t}}$, where $p_{i_{1}}, \ldots, p_{i_{t}}$ are the distinct prime divisors of $n$. If we need to distinguish between the two different types of representation we will refer to the first type as a numerical representation and the second type as a coordinate representation, also called the cellular representation by Evans, Fricke, Maneri, McKee, and Perkel [4]. These two types of representation are equivalent. The conversion of a numerical representation to a coordinate representation has already been described. The conversion of a coordinate representation to a numerical representation requires applying the Chinese remainder theorem.

Nešetřil and Pultr [8] define the dimension of a graph $G$ to be the smallest integer $m$ for which $G$ is isomorphic to an induced subgraph of $K_{n_{1}} \times_{K} \cdots \times_{K} K_{n_{m}}$ for some integers $n_{1}, \ldots, n_{m}$. This is denoted $\operatorname{pdim}(G)$ in West [11] and we will use this notation for dimension. As $\operatorname{pdim}(G)$ is defined for all graphs we obtain the following.

Theorem 2.4 Any graph can be represented modulo a product of some set of distinct primes.

Proof. See Nešetřil and Pultr [8] and pages 376-378 in West [11].

Thus we may think of $\operatorname{pdim}(G)$ as the smallest number of prime factors we can have in a representation of $G$ modulo a product of distinct primes. A related representation parameter is given by Silva [9]. The degree of a representation modulo $n$ is defined to be the number of prime divisors of $n$, counting multiplicities. The representation degree, $d_{r}(G)$, of a graph is the smallest degree of any representation of $G$. Clearly $\operatorname{pdim}(G) \geq d_{r}(G)$, and if $\mathrm{G}$ is reduced then $\operatorname{pdim}(G)=d_{r}(G)$, and $p \operatorname{dim}(G) \leq$ the number of primes in the factorization of $\operatorname{Rep}(G)$.

In computing $d_{r}(G)$ we can use edge labellings of $G^{C}$, the complement of $G$. An edge colouring of a graph $G$ is an assignment of colours to the edges of $G$ so that adjacent edges are coloured differently. The chromatic index of $G$, written $\chi^{\prime}(G)$, is the smallest number of colours needed for an edge colouring of $G$. A transitive edge labelling of $G$ is an assignment of colours to the edges of $G$ so that adjacent edges can be coloured the same only if they form two edges of a triangle, each of whose edges is coloured the same. The transitive degree of $G$, written $d_{t}(G)$ is the smallest number of colours needed for a transitive edge labelling of $G$. As an edge colouring 
is a transitive edge labelling, we see that $d_{t}(G) \leq \chi^{\prime}(G)$. It is easily seen that if $G$ is triangle-free then $d_{t}(G)=\chi^{\prime}(G)$.

Let $\triangle(G)$ denote the maximum degree of a vertex of $G$.

We will make use of the following well-known results on chromatic indices.

Theorem 2.5 Let $G$ be a graph. Then

(i) $\chi^{\prime}(G)=\triangle(G)$ or $\triangle(G)+1$.

(ii) If $G$ is bipartite then $\chi^{\prime}(G)=\triangle(G)$

Proof. See Vizing [10] for a) and Konig [5] for b).

The next result describes the relationship between the dimension and representation degrees of graphs and edge labelling of their complements.

Theorem 2.6 (i) If $\chi^{\prime}\left(G^{C}\right) \geq 2$ then $\operatorname{pdim}(G) \leq \chi^{\prime}\left(G^{C}\right)$, and when $G^{C}$ is trianglefree this becomes an equality.

(ii) If $d_{t}\left(G^{C}\right) \geq 2$ then $d_{r}(G) \leq d_{t}\left(G^{C}\right)$, and when $G^{C}$ is triangle-free this becomes an equality.

Proof. See Proposition 2.3 of Lovász, Nešetřil, and Pultr [6] for (i), and Corollaries 5 and 8 of Silva [9] for (ii).

As, when $G^{C}$ is triangle-free, $d_{t}\left(G^{C}\right)=\chi^{\prime}\left(G^{C}\right)$ the following is immediate.

Theorem 2.7 Suppose that $G^{C}$ is triangle-free, $\triangle\left(G^{C}\right) \geq 2$. Then

(i) $d_{r}(G)=\chi^{\prime}\left(G^{C}\right)=\triangle\left(G^{C}\right)$ or $\triangle\left(G^{C}\right)+1$.

(ii) If $G^{C}$ is bipartite then $d_{r}(G)=\triangle\left(G^{C}\right)$.

Furthermore if $G$ contains an induced subgraph with a triangle-free complement then $d_{r}$ is at least the maximum degree in the triangle-free complement.

Proof. A proof of Theorem 2.7 (ii), not using chromatic indices, for the case in which $G^{C}$ is acyclic, was given by Silva [9]: the stronger result is an observation of Evans $[2]$.

Theorem 2.8 (i) A graph is representable modulo some prime if and only if it is complete.

(ii) A graph is representable modulo a product of some pair of distinct primes if and only if it does not contain an induced subgraph isomorphic to $K_{2}+2 K_{1}, K_{3}+K_{1}$, or the complement of a chordless cycle of length at least five.

Proof. Part (i) is trivial. For part (ii) there are two different proofs in the literature. See Theorem 2.6 in Nešetřil and Pultr [8], or Theorem 4.1 in Evans, Fricke, Maneri, McKee, and Perkel [4]. 
Corollary 2.9 (i) A graph is representable modulo some power of a prime if and only if it is a complete multipartite graph.

(ii) A graph is representable modulo a product of some pair of powers of distinct primes if and only if its reduction does not contain an induced subgraph isomorphic to $K_{2}+2 K_{1}, K_{3}+K_{1}$, or the complement of a chordless cycle of length at least five.

The next theorem extends the part of Theorem 2.8 involving an induced $K_{3}+K_{1}$. It is still unknown if there is a simple extension of the part involving induced $K_{2}+2 K_{1}$ and chordless cycles. For a summary of some other problems involving representation numbers see Evans [3].

Lemma 2.10 Let $G$ be a graph that contains $K_{m}+K_{1}$ as an induced subgraph. If $G$ is representable modulo $n$, then $n$ contains at least $m$ distinct primes.

Proof. This is essentially proved Lovász, Nešetřil, and Pultr [6] we only need to observe that the coordinates of the $K_{m}+K_{1}$ must be distinct. By relabelling we may assume coordinates of the $K_{1}$ vertex are all 0 . Since no two of the $K_{m}$ vertices agree on a coordinate each must agree somewhere with the $K_{1}$ vertex, there must be at least $m$ coordinates.

In Theorem 3.6 we will determine $\operatorname{Rep}\left(K_{m}+K_{1}\right)$.

All of our results so far have dealt with the number of prime divisors of representation numbers. The following result tells us about the possible sizes of these prime factors.

Theorem 2.11 If $G$ is representable modulo $n$ and $p$ is a prime divisor of $n$ then $p \geq \chi(G)$. Thus, if $G$ is reduced then $\operatorname{Rep}(G) \geq p_{i} p_{i+1} \cdots p_{i+m-1}$, where $p_{i}$ is the smallest prime satisfying $p_{i} \geq \chi(G)$ and $m=d_{r}(G)=\operatorname{pdim}(G)$.

Proof. See Theorem 1.2 in Evans, Fricke, Maneri, McKee, and Perkel [4] and Silva [9] and use the fact observed above that $\operatorname{pdim}(G)=d_{r}(G)$ for reduced graphs

Corollary 2.12 If $G$ contains a $K_{m}+K_{1}$ and $p_{i}$ is the smallest prime satisfying $p_{i} \geq \chi(G)$ then $\operatorname{Rep}(G) \geq p_{i} p_{i+1} \cdots p_{i+m-1}$.

Proof. Apply Lemma 2.10.

We also note the following trivial bound which follows because vertex labels are distinct in a representation.

Lemma 2.13 If $G$ is representable modulo $n$ then $G$ has at most $n$ vertices. 


\section{Stars, Paths and Cycles}

In this section we explore representations of paths, cycles and graphs of the form $K_{m}-P_{n}, K_{m}-C_{n}$ and $K_{m}-K_{1, l}$ (graphs whose complements are paths, cycles and stars along with isolated vertices).

For most of our examples in this section we will have $\operatorname{Rep}(G)=p_{i} p_{i+1} \cdots p_{i+m-1}$ where $m=d_{r}(G)$, meeting the bounds in Theorem 2.11 and Corollary 2.12. This will not always be the case for reduced graphs. Consider $K_{n} \times_{K} K_{2}$. We have $d_{r}\left(K_{n} \times K_{2}\right)=2$, but clearly, by Lemma 2.13, if $n>6$ then $\operatorname{Rep}\left(K_{n} \times_{K} K_{2}\right)>2 \cdot 3$.

We begin by stating some basic results on paths and cycles that are known or can be found by combining known results.

Theorem $3.1 \operatorname{Rep}\left(P_{n}\right)=2 \cdot 3 \cdots p_{\left\lceil\log _{2}(n-1)\right\rceil}$.

Proof. The cases where $n=1$ and $n=2$ are trivial and are covered in Theorem 4.1. It is easily checked that $\operatorname{Rep}\left(P_{3}\right)=6$ with representation $\{0,1,2\}$ and $\operatorname{Rep}\left(P_{4}\right)=6$ with representation $\{0,1,2,3\}$.

Lovász, Nešetřil, and Pultr [6] show for $n \geq 3$ that $\operatorname{pdim}\left(P_{n}\right)=\left\lceil\log _{2}(n-1)\right\rceil$. (Note, in [6] $P_{n}$ is a path on $n+1$ vertices where here we use $P_{n}$ for a path on $n$ vertices.) Hence, by Theorem $2.11, \operatorname{Rep}\left(P_{n}\right) \geq 2 \cdot 3 \cdots p_{\left[\log _{2}(n-1)\right]}$. To get equality, for $n \geq 3$, the product dimension representations given in [6] can easily be seen to be coordinate representations modulo $2 \cdot 3 \cdots p_{\left[\log _{2}(n-1)\right]} \cdot \operatorname{Rep}\left(P_{n}\right) \leq 2 \cdot 3 \cdots p_{\left[\log _{2}(n-1)\right\rceil}$ is also shown in Corollary 3.2 in Evans, Fricke, Maneri, McKee,and Perkel [4].

Theorem 3.2 $\operatorname{Rep}\left(C_{4}\right)=4$, and if $n \geq 3, \operatorname{Rep}\left(C_{2 n}\right)=2 \cdot 3 \cdots p_{\left\lceil\log _{2}(n-1)\right\rceil+1}$.

Proof. It is easily checked that $\operatorname{Rep}\left(C_{4}\right)=4$ with representation $\{0,1,2,3\}$.

Lovász, Nešetřil, and Pultr [6] show that $\operatorname{pdim}\left(C_{2 n}\right)=\left\lceil\log _{2}(n-1)\right\rceil+1$ for $n \geq 3$. Hence, by Theorem 2.11, $\operatorname{Rep}\left(C_{2 n}\right) \geq 2 \cdot 3 \cdots p_{\left\lceil\log _{2}(n-1)\right\rceil+1}$, when $n \geq 3$. To get equality, for $n \geq 3$, the product dimension representations given in [6] can easily be seen to be coordinate representations modulo $2 \cdot 3 \cdots p_{\left\lceil\log _{2}(n-1)\right\rceil+1} \cdot \operatorname{Rep}\left(C_{2 n}\right) \leq$ $\left.p_{i} p_{i+1} p \cdots \log _{2}(n-1)\right\rceil+1$ when $n \geq 3$ is also shown by Corollary 3.3 in Evans, Fricke, Maneri, McKee, and Perkel [4].

Theorem $3.3 \operatorname{Rep}\left(C_{5}\right)=3 \cdot 5 \cdot 7=105, \operatorname{Rep}\left(C_{7}\right)=3 \cdot 5 \cdot 7 \cdot 11=1155$, and if $n \geq 4$ and $n$ is not a power of 2 then $\operatorname{Rep}\left(C_{2 n+1}\right)=3 \cdot 5 \cdots p_{\left\lceil\log _{2} n\right\rceil+1}$.

Proof. As noted in [4], it can be checked that $\operatorname{Rep}\left(C_{5}\right)=3 \cdot 5 \cdot 7$ and $\operatorname{Rep}\left(C_{7}\right)=$ $3 \cdot 5 \cdot 7 \cdot 11$.

Lovász, Nešetřil, and Pultr [6] show that $\operatorname{pdim}\left(C_{2 n+1}\right) \geq\left\lceil\log _{2} n\right\rceil+1$ for $n \geq 2$. Hence, by Theorem 2.11, $\operatorname{Rep}\left(C_{2 n+1}\right) \geq 3 \cdot 5 \cdots p_{\left\lceil\log _{2} n\right\rceil+1}$, when $n \geq 2$. To get equality, 
for $n \geq 5, n$ not a power of 2, Theorem 3.2 in Evans, Fricke, Maneri, McKee, and Perkel [4] shows that $\operatorname{Rep}\left(C_{2 n+1}\right) \leq 3 \cdot 5 \cdots p_{\left\lceil\log _{2} n\right\rceil+1}$.

Note that the result of Evans, Fricke, Maneri, McKee, and Perkel [4] above shows that when $n$ is not a power of $2, \operatorname{pdim}\left(C_{2 n+1}\right)=\left\lceil\log _{2} n\right\rceil+1$. This had been left as a question in remark 6.4 of Lovász, Nešetrril, and Pultr [6]. For the remaining cases, $C_{2 n+1}$ where $n$ is of the form $n=2^{s}$ both the product dimension and the representation number are unknown. However in [6], it is shown that for these cases $\left\lceil\log _{2} n\right\rceil+1 \leq p \operatorname{dim}\left(C_{2 n+1}\right) \leq\left\lceil\log _{2} n\right\rceil+2$ and the upper bound holds for $n$ of the form $2^{2^{t+1}}$.

Next we consider cases involving graphs whose complement is a star, path or cycle along with isolated vertices. Here, addition of isolated vertices does not seem to cause problems, but in the cases of paths and cycles from above and stars (considered in Section 5) addition of isolated vertices seems to make the problem of determining the representation number much more difficult.

The graph $K_{m}-P_{2}$ is not reduced and we will see in Section 5 that $\operatorname{Rep}\left(K_{m}-P_{2}\right)=$ $p_{s}^{2}$ where $p_{s}$ is the smallest prime greater than or equal to $m-1$.

Theorem 3.4 If $m \geq n \geq 3$, then $\operatorname{Rep}\left(K_{m}-P_{n}\right)=p_{i} p_{i+1}$ where $p_{i}$ is the smallest prime greater than or equal to $m-n+\left\lceil\frac{n}{2}\right\rceil$.

Proof. Note that $G^{C}$ is a path on $n$ vertices union a set of $m-n$ isolated vertices. $K_{m}-P_{n}$ for $n \geq 3$ contains a $K_{2}+K_{1}$ and a clique of size $m-n+\left\lceil\frac{n}{2}\right\rceil$. So by Corollary 2.12, $p_{i} \geq m-n+\left\lceil\frac{n}{2}\right\rceil$ and $\operatorname{Rep}\left(K_{m}-P_{n}\right) \geq p_{i} p_{i+1}$.

Next we show $K_{m}-P_{n}$ is representable modulo $p_{i} p_{i+1}$. We give a coordinate representation with respect to $p_{i}$ and $p_{i+1}$ to the vertices of $K_{m}-P_{n}$ as follows. Let $v_{1}, \ldots, v_{n}$ be the vertices of the 'removed path'. Assign coordinates $\left(\left\lfloor\frac{i-1}{2}\right\rfloor,\left\lceil\frac{i-1}{2}\right\rceil\right)$ to $v_{i}$ for $i=1, . ., n$. The remaining $m-n$ vertices of $K_{m}-P_{n}$ will be assigned coordinates $\left(i+\left\lceil\frac{n+1}{2}\right\rceil, i+\left\lceil\frac{n+1}{2}\right\rceil\right)$ for $i=0, \ldots, m-n$.

The graph $K_{m}-C_{3}$ is not reduced and we will see in Section 5 that $\operatorname{Rep}\left(K_{3}-C_{3}\right)=$ $2 \cdot 3$, that $\operatorname{Rep}\left(K_{4}-C_{3}\right)=2^{3}$ and that for $m \geq 5, \operatorname{Rep}\left(K_{m}-C_{3}\right)=p_{s}^{2}$ where $p_{s}$ is the smallest prime greater than or equal to $m-2$.

Theorem 3.5 If $m \geq n \geq 4$, let $p_{i}$ be the smallest prime greater than or equal to $m-n+\left\lceil\frac{n}{2}\right\rceil$. Then

(i) $\operatorname{Rep}\left(K_{m}-C_{n}\right)=p_{i} p_{i+1}$ if $n$ is even.

(ii) $\operatorname{Rep}\left(K_{m}-C_{n}\right)=p_{i} p_{i+1} p_{i+2}$ if $n$ is odd.

Proof. Note that $G$ is reduced and that $G^{C}$ is a cycle on $n$ vertices union a set of $m-n$ isolated vertices. So $G^{C}$ is triangle free with $\chi^{\prime}\left(G^{C}\right)=2$ if $n$ is even and $\chi^{\prime}\left(G^{C}\right)=3$ if $n$ is odd. Thus by Theorem $2.7, d_{r}(G)=2$ if $n$ is even and 3 if $n$ is 
odd. Further $\chi(G)=m-n+\left\lceil\frac{n}{2}\right\rceil$. Thus, by Corollary 2.12, $\operatorname{Rep}(G) \geq p_{i} p_{i+1}$ if $n$ is even and $\operatorname{Rep}(G) \geq p_{i} p_{i+1} p_{i+2}$ if $n$ is odd, where $p_{i}$ is the smallest prime satisfying $p_{i} \geq m-n+\left\lceil\frac{n}{2}\right\rceil$.

For equality in (i) We use the same labelling as in Theorem 3.4 with the exception that the vertex $v_{n}$ is labelled $\left(\frac{n-1}{2}, 0\right)$ with respect to $p_{i}$ and $p_{i+1}$. This gives us a representation modulo $p_{i} p_{i+1}$.

For equality in (ii) We need only show that $G$ can be represented modulo $p_{i} p_{i+1} p_{i+2}$. To do this we will assign coordinates, with respect to $p_{i}, p_{i+1}, p_{i+2}$, to the vertices of $K_{m}-C_{n}$ as follows. Let $v_{1}, \ldots, v_{n}$ be the vertices of the 'removed' cycle. Assign coordinates $\left(\left\lfloor\frac{i}{2}\right\rfloor,\left\lfloor\frac{i+1}{2}\right\rfloor-1,\left\lfloor\frac{i+2}{2}\right\rfloor-1\right)$ to $v_{i}$ for $i=1, \ldots, n-1$, and coordinates $\left(\frac{n-1}{2}, 0, \frac{n-1}{2}\right)$ to $v_{n}$. The remaining $m-n$ vertices of $K_{m}-C_{n}$ will be assigned coordinates $(j, j, j)$ for $j=\frac{n+1}{2}, \ldots, m-\frac{n+1}{2}$. It is routine to check that this is a coordinate representation of $G$ modulo $p_{i} p_{i+1} p_{i+2}$.

Stars are complete bipartite graphs and considered in Section 5. Here we consider complements of stars. If $m=n+1$ in the following theorem we get $\operatorname{Rep}\left(K_{n}+K_{1}\right)=$ $p_{s} p_{s+1} \cdots p_{s+n-1}$ where $p_{s}$ is the smallest prime greater than or equal to $n$, since $K_{m}-K_{1, n}=K_{m-n+1} \vee\left(K_{n}+K_{1}\right)$. In Theorem 5.2 we will look at $\operatorname{Rep}\left(K_{n}+t K_{1}\right)$.

Theorem 3.6 If $n \leq m-1$ then $\operatorname{Rep}\left(K_{m}-K_{1, n}\right)=p_{s} p_{s+1} \cdots p_{s+n-1}$ where $p_{s}$ is the smallest prime greater than or equal to $m-1$.

Proof. Note that $G^{C}$ is star on $n+1$ vertices union a set of $m-n+1$ isolated vertices. By Corollary 2.12, a clique of size $m-1$ and an induced $K_{n}+K_{1}$ imply $\operatorname{Rep}(G) \geq p_{s} p_{s+1} \cdots p_{s+n-1}$.

We will give a coordinate representation modulo $p_{s} p_{s+1} \cdots p_{s+n-1}$. Let $v_{1}, \ldots, v_{m-n-1}$

be the vertices not in the star in $G^{C}, v_{m-n}$ be the root of the star, and $v_{m-n+1}, \ldots, v_{m}$ be the vertices in the star. Vertices $v_{j}, 1 \leq j \leq m-n$ will be labelled with the coordinates $(j-1, \ldots, j-1)$. Vertices $v_{m-n+i}, 1 \leq i \leq n$ will be labelled with the coordinates as follows. For a fixed $i$, the first $i-1$ coordinate entries equal $m-n+i-2$, the $i$-th coordinate entry is equal to $m-n-1$, and the remaining coordinate entries are equal to $m-n+i-1$. The above yields a representation modulo $p_{s} p_{s+1} \cdots p_{s+n-1}$.

\section{Complete Graphs}

In this section we consider disjoint unions of complete graphs and the relationship of their representation number to mutually orthogonal Latin squares. Complements of disjoint unions of complete graphs are complete multipartite graphs and will be considered in Section 5.

We begin by reviewing some easy results. 
Theorem 4.1 Rep $\left(K_{m}\right)$ is the smallest prime greater than or equal to $\mathrm{m}$.

Proof. This is proved in Evans, Fricke, Maneri, McKee, and Perkel [4]. The lower bound follows from Theorem 2.11 and $\{0,1,2, \ldots, m-1\}$ is a representation modulo this prime.

The graph $n K_{1}$ of isolated vertices is not reduced. As discussed in Section 5 , it is easy to see that $\operatorname{Rep}\left(n K_{1}\right)=2 n$.

The next result on matchings is easily obtained from product dimension results.

Theorem 4.2 $\operatorname{Rep}\left(n K_{2}\right)=2 \cdot 3 \cdots p_{\left\lceil\log _{2} n\right\rceil+1}$.

Proof. Lovász, Nešetřil, and Pultr [6] show that $\operatorname{pdim}\left(n K_{2}\right)=\left\lceil\log _{2} n\right\rceil+1$. Hence by Theorem $2.11, \operatorname{Rep}\left(n K_{2}\right) \geq 2 \cdot 3 \cdots p_{\left\lceil\log _{2} n\right\rceil+1}$. To get equality, we can easily get a coordinate representation by taking a binary sequence and its complement for the endpoints of each edge, making sure that the choices are distinct (see West [11, page $378])$.

Next suppose that $G=n K_{m}$, where $n, m \geq 2$. $n K_{m}$ is reduced and $\chi\left(n K_{m}\right)=$ $m$ and $n K_{m}$ contains an induced $K_{m}+K_{1}$, so by Corollary $2.12, \operatorname{Rep}\left(n K_{m}\right) \geq$ $p_{i} p_{i+1} \cdots p_{i+m-1}$, where $p_{i}$ is the smallest prime satisfying $p_{i} \geq m$. When we get equality is related to the existence of orthogonal Latin squares.

Theorem 4.3 If $n, m \geq 2$ then $\operatorname{Rep}\left(n K_{m}\right)=p_{i} p_{i+1} \cdots p_{i+m-1}$, where $p_{i}$ is the smallest prime satisfying $p_{i} \geq m$, if and only if there exists a set of $n-1$ mutually orthogonal Latin squares of order $m$.

Proof. We have already observed that $\operatorname{Rep}\left(n K_{m}\right) \geq p_{i} p_{i+1} \cdots p_{i+m-1}$. So we need to show equality.

Let $L_{1}, L_{2}, \ldots, L_{n-1}$ be a set of mutually orthogonal Latin squares of order $m$ with entries $0,1, \ldots, m-1$ and rows and columns indexed by $1,2, \ldots, m$. The row $k$ column $j$ entry of $L_{i}$ will be denoted $L_{i}(k, j)$ Let the vertices of $G=n K_{m}$ be denoted $v(i j), i=1,2, \ldots, n, j=0,1, \ldots, m-1$ with $v(i j)$ adjacent to $v\left(i^{\prime} j^{\prime}\right)$ if and only if $i=i^{\prime}$ and $j \neq j^{\prime}$.

The coordinate representation $\left(v_{1}(i j), v_{2}(i j), \ldots, v_{m}(i j)\right)$ of $v(i j)$ is specified as follows. The coordinate representation of $v(1 j)$ is $(j-1, j-1, \ldots, j-1)$. That is, $v_{k}(1 j)=j-1$ for $j=1,2, \ldots, m$ and $k=1,2, \ldots, m$. The coordinate representation of $v(2 j)$ is equal to the $j^{\text {th }}$ column of $L_{1}$. That is, $v_{k}(2 j)=L_{1}(k, j)$ for $j=1,2, \ldots, m$ and $k=1,2, \ldots, m$. For $i=3,4 \ldots, n$ and $j=1,2, \ldots, m$ and $k=1,2, \ldots, m$ we have $v_{k}(i j)=L_{1}(k, x)$ where $L_{i-1}(k, x)=j-1$. So, to determine the coordinate representation of $v(i j)$ for $i \geq 3$, find the locations of the entry $j-1$ in the $(i-$ $1)^{s t}$ Latin square, 'superimposing' these locations on the first Latin square gives a transversal. Read these entries from top to bottom. 
Using elementary properties of orthogonal Latin squares it is straightforward to check that this is a representation. For completeness we include the details.

- $v(1 j)$ is adjacent to $v\left(1 j^{\prime}\right)$ for $j \neq j^{\prime}$ since $(j-1, j-1, \ldots, j-1)$ and $\left(j^{\prime}-1, j^{\prime}-\right.$ $\left.1, \ldots, j^{\prime}-1\right)$ disagree everywhere.

- $v(2 j)$ is adjacent to $v\left(2 j^{\prime}\right)$ for $j \neq j^{\prime}$ since the entries of a row of a Latin square are distinct. Hence two columns disagree on each coordinate.

- $v(i j)$ is adjacent to $v\left(i j^{\prime}\right)$ for $i=3,4, \ldots, n$ and $j \neq j^{\prime}$ since the locations of the entries $j-1$ and the entries $j^{\prime}-1$ in $L_{i-1}$ are disjoint and since the rows of $L_{1}$ have distinct entries.

- $v(1 j)$ is not adjacent to $v\left(2 j^{\prime}\right)$ since the coordinates for $v\left(2 j^{\prime}\right)$ form a permutation of $0,1, \ldots, m-1$ as they correspond to a column of $L_{2}$ and $v(1 j)$ has representation $(j-1, j-1, \ldots j-1)$ where $j \in\{0,1, \ldots, m-1\}$.

- $v(1 j)$ is not adjacent to $v\left(i j^{\prime}\right)$ for $i=3,4, \ldots, n$ since the coordinates of $v\left(i j^{\prime}\right)$ form a permutation of $0,1, \ldots, m-1$ as they correspond to a transversal in $L_{1}$ (it is a transversal since $L_{1}$ and $L_{i-1}$ are orthogonal) and $v(1 j)$ has representation $(j-1, j-$ $1, \ldots j-1)$ where $j \in\{0,1, \ldots, m-1\}$. Thus the two agree on one coordinate.

- $v(2 j)$ is not adjacent to $v\left(i j^{\prime}\right)$ for $i=3,4, \ldots, n$ since the coordinates of $v\left(i j^{\prime}\right)$ are from a transversal of $L_{1}$ and the coordinates of $v(2 j)$ are from a column of $L_{1}$ and each transversal in $L_{1}$ intersects each column. Thus the two agree on one coordinate. - $v(i j)$ is not adjacent to $v\left(i^{\prime} j^{\prime}\right)$ for $i, i^{\prime} \in\{3,4, \ldots, n\}$ with $i \neq i^{\prime}$. Since $L_{i-1}$ and $L_{i^{\prime}-1}$ are orthogonal, the positions of the entries $j-1$ in $L_{i-1}$ and $j^{\prime}-1$ in $L_{i^{\prime}-1}$ agree in one location and hence the corresponding transversals in $L_{1}$ intersect. That is, for some $k$ and $x, L_{i-1}(k, x)=j-1$ and $L_{i^{\prime}-1}(k, x)=j^{\prime}-1$, then $v_{k}(i j)=v_{k}\left(i^{\prime} j^{\prime}\right)=L_{1}(k, x)$.

For the converse, assume $\operatorname{Rep}\left(n K_{m}\right)=p_{i} p_{i+1} \cdots p_{i+m-1}$ and we have a coordinate representation of $n K_{m}$ meeting this bound. For each $k=1,2, \ldots, m$ we may relabel the entries $v_{k}(i j)$ without changing the representation number. Also for each $k$, the entries $v_{k}(11), v_{k}(12), \ldots, v_{k}(1 m)$ must be distinct since $v(11), v(12), \ldots, v(1 m)$ is a clique. Thus after relabeling we may assume that $v_{k}(1 j)=j-1$. That is, the coordinate representation of $v(1 j)$ is $(j-1, j-1, \ldots, j-1)$. Then, since each $v(i j)$ for $i=2,3, \ldots, n, j=0,1, \ldots, m-1$ is not adjacent to all of $v(11), v(12), \ldots, v(1 m)$, the coordinate representation of $v(i j)$ must be a permutation of $\{0,1, \ldots, m-1\}$ in order for all to agree on at least on coordinate with each of $v(11), v(12), \ldots, v(1 m)$. Also, the permutations for $v(i j)$ and $v\left(i j^{\prime}\right)$ must disagree on all coordinates and those for $v(i j)$ and $v\left(i^{\prime} j^{\prime}\right)$ must agree on exactly one coordinate because of the adjacencies in $n K_{m}$. Then it is straightforward to check that the process described above to get a representation from Latin squares can be reversed to form orthogonal Latin squares.

For those graphs $n K_{m}$ that do not satisfy the conditions of Theorem 4.3 we conjecture the following. 
Conjecture 1 If $n, m \geq 2$ then $\operatorname{Rep}\left(n K_{m}\right)=p_{i} p_{i+1 \ldots} p_{i+t-1}$, where $p_{i}$ is the smallest prime satisfying $p_{i} \geq t$, and $t$ is the smallest integer, $t \geq m$, for which there exists $a$ set of $n-1$ mutually orthogonal Latin squares of order $t$.

Our result on representation numbers of disjoint unions of complete graphs can be used to get an analogue for product dimension.

Corollary 4.4 If $n \leq m, \operatorname{pdim}\left(n K_{m}\right)=m$ if and only if there exist $n-1$ mutually orthogonal Latin squares of order $n$.

Proof. From the theorem we need to show that $\operatorname{pdim}\left(n K_{m}\right)=m$ if and only if $\operatorname{Rep}\left(n K_{m}\right)=p_{i} p_{i+1} \cdots p_{i+m-1}$ where $p_{i}$ is the smallest prime satisfying $p_{i} \geq m$. We will show the more general result that for non-empty $G$ such that $K_{m}+G$ is reduced, $\operatorname{pdim}\left(K_{m}+G\right)=m$ if and only if $\operatorname{Rep}\left(K_{m}+G\right)=p_{i} p_{i+1} \cdots p_{i+m-1}$ where $p_{i}$ is the smallest prime satisfying $p_{i} \geq m$.

By Corollary 2.12 and the analogue for dimension (proposition 3.4 in Lovász, Nešetřil, and Pultr [6]), $\operatorname{pdim}\left(n K_{m}\right) \geq m$ and $\operatorname{Rep}\left(n K_{m}\right) \geq p_{i} p_{i+1} \cdots p_{i+m-1}$. Given either a representation for dimension or a coordinate modular representation with $m$ coordinates, we may relabel the entries within each coordinate and thus may assume that the representations of the vertices of $K_{m}$ are $(0,0, \ldots, 0),(1,1, \ldots, 1), \cdots,(m-$ $1, m-1, \ldots, m-1)$. Then since every vertex in $G$ is non-adjacent to all of the vertices of $K_{m}$ it must agree with each of these vertices on at least one and hence exactly one coordinate. So the coordinate representations of the other vertices are permutations of $\{0,1,2, \ldots, m-1\}$ and thus a modular coordinate representation is a dimension representation and vice versa. We use $p_{i} \geq m$ to ensure that the dimension representation is a modular representation and we use $K_{m}+G$ reduced to ensure that the representations are distinct and thus the modular representation is a dimension representation.

\section{Representation Numbers of Non-Reduced Graphs}

In this section we examine representations of some non-reduced graphs. In particular we look at complete multipartite graphs and complete graphs union isolated vertices. We no longer have the close connection to product dimension that we had for the reduced graphs of the previous sections. If $G$ is a complete multipartite graph then $\operatorname{pdim}(G)=2$, but even for stars determining the representation number is complicated.

Theorem 5.1 $\operatorname{Rep}\left(m K_{1}\right)=2 m$. 
Proof. This proof is from Evans, Fricke, Maneri, McKee, and Perkel [4]. $\{0,2,4, \ldots, 2(m-1)\}$ is a representation modulo $2 m$. A representation modulo some integer less than $2 m$ would imply that two labels would differ by 1 (by the pigeonhole principle), and thus those vertices would be adjacent, a contradiction.

Theorem 5.2 If $t \leq(m-1)$ ! then $\operatorname{Rep}\left(K_{m}+t K_{1}\right)=p_{s} p_{s+1} \cdots p_{s+m-1}$ where $p_{s}$ is the smallest prime greater than or equal to $m$.

Proof. This is essentially shown in proposition 3.6 of Lovász, Nešetřil, and Pultr [6]. By Corollary $2.12 \operatorname{Rep}\left(K_{m}+t K_{1}\right) \geq p_{s} p_{s+1} \cdots p_{s+m-1}$. We will give a coordinate representation. Let $v_{1}, \ldots, v_{m}$ be the vertices of $K_{m}$, and $v_{m+1}, \ldots, v_{m+t}$ be the remaining vertices. Assign coordinates $(j-1, \ldots, j-1)$ to $v_{j}$ for $1 \leq j \leq m$, and $\left(0, s_{1}, s_{2}, \ldots, s_{m-1}\right)$ to $v_{j}$ for $m+1 \leq j \leq m+t$ where each string $\left\{s_{1}, s_{2}, \ldots, s_{m-1}\right\}$ is a different permutation of the numbers $1, . ., m-1$.

We now see how the addition of even a single isolated vertex can make determining the representation number complicated.

Corollary 5.3 There exist graphs $G$ where $\operatorname{Rep}\left(G+K_{1}\right)=\operatorname{Rep}(G)$ and where $\operatorname{Rep}\left(G+K_{1}\right)-\operatorname{Rep}(G)$ is arbitrarily large.

Proof. The first part is trivial since Theorem 5.2 implies $\operatorname{Rep}\left(K_{m}+K_{1}\right)=\operatorname{Rep}\left(K_{m}+\right.$ $\left.(m-1) ! K_{1}\right)$.

For the second part, by Theorem 4.1, $\operatorname{Rep}\left(K_{m}\right)=p_{s}$ where $p_{s}$ is the smallest prime greater than or equal to $m$. By Theorem 5.2, $\operatorname{Rep}\left(K_{m}+K_{1}\right)=p_{s} p_{s+1} \cdots p_{s+m-1}$. Hence we can make the difference $\operatorname{Rep}\left(G+K_{1}\right)-\operatorname{Rep}(G)$ for $G=K_{m}$, arbitrarily large by simply choosing $m$ large enough.

In Theorem $5.2 t=(m-1)$ ! is best possible in that $\operatorname{Rep}\left(K_{m}+t K_{1}\right)>\operatorname{Rep}\left(K_{m}+\right.$ $\left.(m-1) ! K_{1}\right)$ for $t>(m-1)$ !. This follows from proposition 3.6 of Lovász, Nešetřil, and Pultr [6], stating that $\operatorname{pdim}\left(K_{m}+t K_{1}\right)=m+1$. The observation is that as in the proof of Theorem 5.2 the vertices of the $t K_{1}$ must be a permutation of $\{0,1, \ldots, m-1\}$ and each of these permutations viewed as a coordinate representation must share a coordinate. It is easy to see that there are at most $(m-1)$ ! such 'intersecting' permutations.

While the product dimension of $K_{m}+t K_{1}$ is $m+1$ for all $t>(m-1)$ ! determining the representation number for such graphs is not straightforward. By 'reversing' the process of reduction we can get some partial results, which depend in part on the distribution of particular primes.

Corollary 5.4 Let $p_{s}$ be the smallest prime greater than or equal to $m$. Then Rep $\left(K_{m}+\right.$ $\left.t K_{1}\right)=p_{s}^{2} p_{s+1} \cdots p_{s+m-1}$ for $(m-1) !+1 \leq t \leq p_{s}(m-1) !$. Furthermore, if $p_{s+i-1}(m-1) !+1 \leq t \leq p_{s+i}(m-1)$ ! and $p_{s+m-1}<p_{s}^{2}$ then $\operatorname{Rep}\left(K_{m}+t K_{1}\right)=$ $p_{s} p_{s+1} \cdots p_{s+i}^{2} \cdots p_{s+m-1}$. 
Proof. As noted above, $\operatorname{Rep}\left(K_{m}+t K_{1}\right)>p_{s} p_{s+1} \cdots p_{s+m-1}$.

Using the same coordinate representation from the proof of Theorem 5.2 we can represent $K_{m}+t K_{1}$ modulo $p_{s} p_{s+1} \cdots p_{s+i}^{2} \cdots p_{s+m-1}$ for $t \leq p_{s+i}(m-1)$ ! by placing $p_{s+i}$ vertices in each cell (i.e., allowing $p_{s+i}$ vertices to have the same coordinates) as in the comments after Theorem 2.3.

It remains to show that there is no representation modulo a smaller number $n$ in each case. By Lemma 2.10 and Theorem $2.11 n$ must have at least $m$ distinct primes all of which are greater than or equal to $p_{s}$. So the next smallest possibility is $p_{s}^{2} p_{s+1} \cdots p_{s+m-1}$ and thus the result holds when $t \leq p_{s}(m-1)$ !. For representation modulo $p_{s} p_{s+1} \cdots p_{s+i}^{2} \cdots p_{s+m-1}$ when $i \geq 1$, the only smaller possible values of $n$ are $p_{s} p_{s+1} \cdots p_{s+j}^{2} \cdots p_{s+m-1}$ with $j<i$, using the condition $p_{s+m-1}<p_{s}^{2}$ to eliminate possibilities like $p_{s}^{3} p_{s+1} \cdots p_{s+m-1}$.

In these cases there can be at most $p_{s+j}$ vertices with each coordinate representation and as noted before the proof each coordinate representation is a permutation of $\{0,1, \ldots, m-1\}$. So, if $t \geq p_{s+i}(m-1) !+1>p_{s+j}(m-1)$ !, we would have more than $(m-1)$ ! permutations for which each pair agrees on some coordinate, which is impossible.

In the previous proof we had several isolated vertices using the same coordinate representation. We could also do this for the vertices of the clique. For example, the graph $H+p_{s+i}(m-1) ! K_{1}$ where $H$ is the complete multipartite graph with $m$ parts of size $p_{s+i}$ can be represented modulo $p_{s} p_{s+1} \cdots p_{s+i}^{2} \cdots p_{s+m-1}$ as it can be reduced to $K_{m}+(m-1) ! K_{1}$ by identifying independent sets of size $p_{s+i}$ with the same neighbourhood.

We have referred several times in the previous sections to graphs that are complete multipartite graphs. Complements of disjoint unions of complete graphs are complete multipartite graphs, as well as the special cases $K_{m}-P_{2}$ and $K_{m}-C_{3}$. Each of these last two fits into a special type of complete multipartite graphs, $K_{m}-K_{n}=$ $K_{m-n} \vee n K_{1}$ which have $n$ parts of size 1 and one part of size $m-n$. Even for this case it seems difficult to determine the representation number.

Recall Corollary 2.9(i) which says that $G$ is representable modulo a prime power if and only if it is a complete multipartite graph. However, as we shall see, the smallest $n$ representing such a graph will not always be a prime power. We begin with a general result on multipartite graphs.

Theorem 5.5 The complete multipartite graph $K_{s_{1}, s_{2}, \ldots, s_{m}}$ is representable modulo $\Pi_{i=1}^{d} p_{i}^{k_{i}}$ if and only if $p_{i} \geq m$ for $i=1,2, \ldots, d$ and if for $C=\prod_{i=1}^{d} p_{i}^{k_{i}-1}$ there exist $y_{i j}, i=1,2, \ldots, m, j=1,2, \ldots, d$ such that $C \sum_{j=1}^{d} y_{i j} \geq s_{i}$ for $i=1,2, \ldots, m$ and $\sum_{i=1}^{m} y_{i j} \leq p_{j}$ for $j=1,2, \ldots, d$. 
Proof. This follows almost immediately from the view of the coordinate representation in the paragraph following Theorem 2.3. Each coordinate represents at most $C$ vertices and the coordinates representing the independent vertices in a part can be assumed to form a subarray. The number of vertices representable in the subarray must be at least the size of the part, hence the condition $C \sum_{j=1}^{d} y_{i j} \geq s_{i}$ and the subarrays must be 'disjoint' in the sense that they do not share a coordinate, hence the condition $\sum_{i=1}^{m} y_{i j} \leq p_{j}$. That the $p_{i} \geq m$ follows from Theorem 2.11.

It is not difficult to see that in the previous theorem we make assume some $y_{i j}=1$ for each $i$. That is, the cells for the vertices in each part can be taken to lie in some lower dimensional subarray.

An easy corollary follows.

Corollary 5.6 Let $p_{s}$ be the smallest prime greater than or equal to $m$. If $G$ is a complete multipartite graph with $m$ parts each of size at most $p_{s}$, then $\operatorname{Rep}(G)=p_{s}^{2}$.

This gives the representation number for graphs mentioned in the previous section: $\operatorname{Rep}\left(K_{m}-P_{2}\right)=p_{s}^{2}$ where $p_{s}$ is the smallest prime greater than or equal to $m-1$ since $K_{m}-P_{2}$ is a complete multipartite graph with one part of size two and $m-2$ parts of size one. For $m \geq 4, \operatorname{Rep}\left(K_{m}-C_{3}\right)=p_{s}^{2}$ where $p_{s}$ is the smallest prime greater than or equal to $m-2$; and $\operatorname{Rep}\left(K_{3}-C_{3}\right)=2 \cdot 3, \operatorname{Rep}\left(K_{4}-C_{3}\right)=2^{3}$ since $K_{m}-C_{3}$ is a complete multipartite graph with one part of size three and $m-3$ parts of size one.

We note the multipartite classes must be kept small. If the cardinality of a class is strictly greater than $p_{s}$ then $\operatorname{Rep}(G)$ will not equal $p_{s}^{2}$.

Even for the case of stars, a general result depends on the distribution of primes. We immediately get the following Corollary, which is noted in [4].

Corollary $5.7 \operatorname{Rep}\left(K_{1, m}\right) \leq \min \left\{2^{\left\lceil\log _{2} m\right\rceil+1}, 2 p\right\}$ where $p$ is the smallest prime greater than or equal to $m+1$.

The comment in [4] also includes the term $3^{\left\lceil\log _{3} m\right\rceil+1}$. However, using the fact that for $m \geq 25$ there exists a prime between $m$ and $6 m / 5$ [7], we see that there always exists a prime $p \geq m+1$ such that $2 p \leq 3^{\left\lceil\log _{3} m\right\rceil+1}$. So the representation number of $K_{1, m}$ will never be a power of 3 . Similar conclusions can be reached for complete multipartite graphs of the form $K_{m}-K_{n}$ where every part except one has size one if the 'large' part is large enough. For complete bipartite graphs that are not stars such terms can be the minimum as $\operatorname{Rep}\left(K_{9,9}\right)=3^{3}$.

Both of the other terms in the corollary can occur as the minimum. It is easy to check that $\operatorname{Rep}\left(K_{1,4}\right)=2^{3}$ and that $\operatorname{Rep}\left(K_{1,5}\right)=2 \cdot 7$. 
It may be possible that $\operatorname{Rep}\left(K_{1, m}\right)$ will not be one of the forms mentioned in the corollary. For example, if there exist primes $p$ and $q$ such that there is no prime and no power of 2 between $(p-1)(q-1)$ and $p q$ then $K_{1,(p-1)(q-1)}$ is representable modulo $2 p q$ and its representation number will either be $2 p q$ or some term involving even more primes.

\section{References}

[1] P. Erdős and A.B. Evans, Representations of graphs and orthogonal Latin square graphs, J. Graph Theory 13 (1989) 593-595.

[2] A.B. Evans, Review of 'Integral representations of graphs, by F.C. Silva', Math Reviews 97f:05175.

[3] A.B. Evans, 'Representations of graphs modulo $n$ : some problems,' submitted.

[4] A.B. Evans, G.H. Fricke, C.C. Maneri, T.A. McKee, M. Perkel, Representation of graphs modulo n, J. Graph Theory 18 (1994) 801-815.

[5] D. Konig, Uber graphen und ihre anwendung auf determinantentheorie und mengenlehre, Math. Ann. 77 (1916) 453-465.

[6] L. Lovász, J. Nešetřil, and A. Pultr, On a product dimension of graphs , J. Combinat. Theory B 29 (1980) 47-67.

[7] J. Nagura, On the interval containing at least one prime number, Proc. Japan. Acad. 28 (1952) 177-181.

[8] J. Nešetřil and A. Pultr, A Dushnik-Miller type dimension of graphs and its complexity, in: Lecture Notes in Computer Science 56, Fundamentals of computation theory (Springer-Verlag, Berlin, 1977) 482-493.

[9] F.C. Silva, Integral representations of graphs, Portugaliae Mathematica 53 (1996) 137-142.

[10] V.G. Vizing, On an estimate of the chromatic class of a p-graph, Diskret Analiz. 3 (1964) 25-30.

[11] D.B. West, Introduction to graph theory (Prentice-Hall 1996). 